This item was submitted to Loughborough's Institutional Repository (https://dspace.lboro.ac.uk/) by the author and is made available under the following Creative Commons Licence conditions.

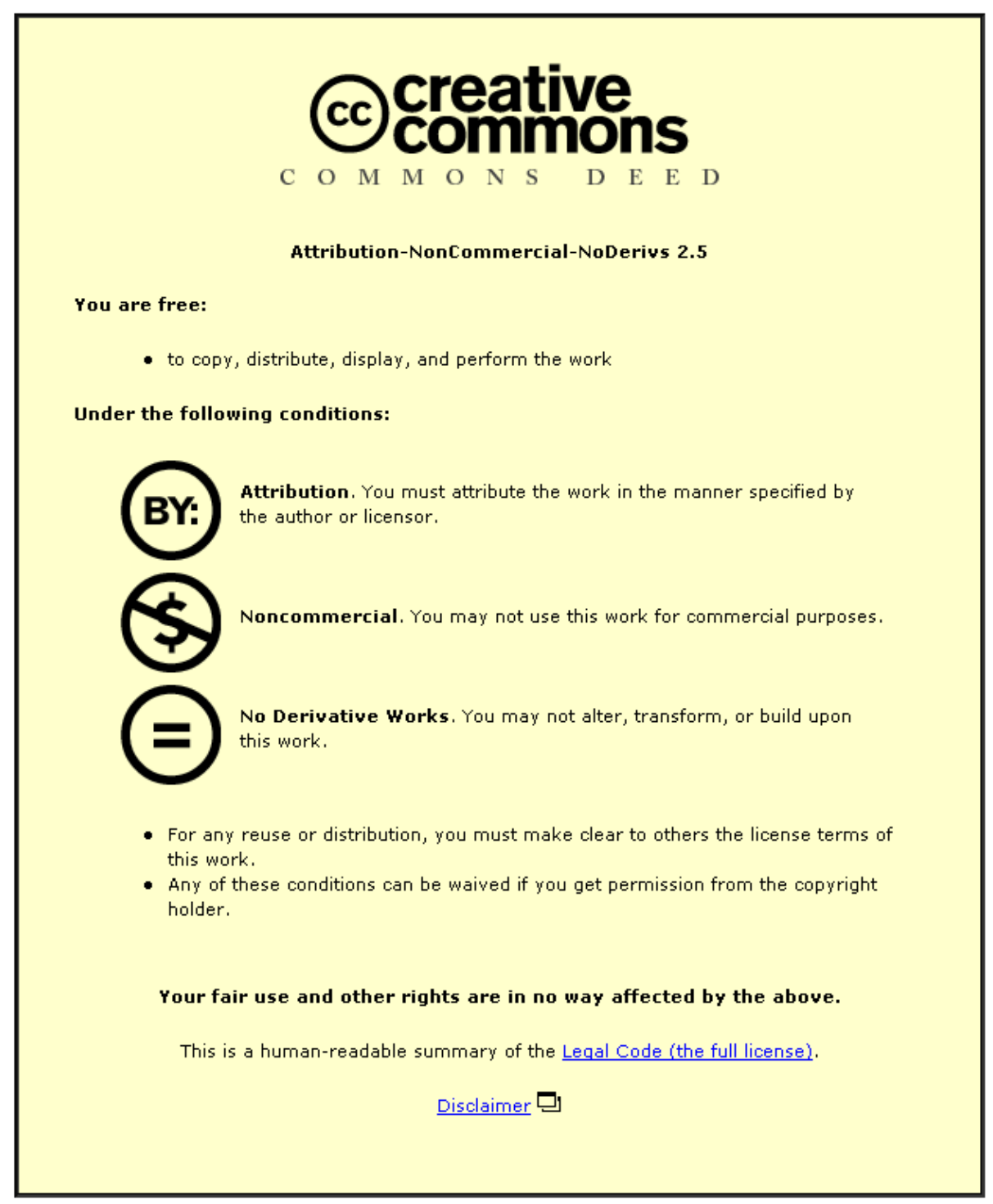

For the full text of this licence, please go to: http://creativecommons.org/licenses/by-nc-nd/2.5/ 


\title{
POST-BUCKLING OF SINGLE AND MULTI-BAY PANELS USING STRUT, STRIP AND FE METHODS
}

\author{
M. Lillico ${ }^{*}$, R. Butler ${ }^{\dagger}$ and G.W. Hunt ${ }^{\ddagger}$ \\ Department of Mechanical Engineering, University of Bath, United Kingdom, BA2 7AY.
}

\author{
A. Watson ${ }^{\S}$ and D. Kennedy ${ }^{\text {Il }}$ \\ Cardiff School of Engineering, Cardiff University, United Kingdom, CF24 0YF.
}

\begin{abstract}
A compressively-loaded stiffened panel has been designed with post-buckling reserve of strength in its skins using the strip program VICONOPT. Comparisons are made with Finite Element single and double bay models. The panel has been shown by VICONOPT to have a stiffener buckling failure mode when an overall sinusoidal imperfection causing increased stiffener compression is present. Such a failure is not seen in a typical single-bay Finite Element model of such panels. However, the failure is confirmed by a double-bay Finite Element model, which is a closer representation of an aerospace application, such as a wing box. A new strut model, following a Shanley type approach has been developed to emphasise the difference between the single and multi-bay responses. The strut model shows good agreement with single bay Finite Element results. It also indicates the presence of an imperfection in the double bay Finite Element model, which may be represented by offsetting the load towards the skin in the strut model. The VICONOPT code is able to design an aerospace panel of realistic dimensions and loading but only when considering linear elastic material properties.
\end{abstract}

\section{Introduction}

It is well known that stiffened panels, used extensively within the aerospace industry, can have a considerable post-buckling reserve of strength in their skins, enabling them to remain in stable equilibrium under loads in excess of their critical local buckling load. By allowing such designs to buckle in a stable manner, for example between limit and ultimate design loads, it is possible to save a significant amount of mass compared to designs for which buckling is not permitted.

Many different techniques have been developed to model the buckling and post-buckling behaviour of

\footnotetext{
Research Officer

$\uparrow$ Senior Lecturer in Aerospace Structures

* Professor of Structural Mechanics

$\S$ Research Associate

II Senior Lecturer

Copyright (C) 2001 by University of Bath and Cardiff University. Published by the American Institute of Aeronautics and Astronautics, Inc. with permission.
}

stiffened panels. These range from finite-strip ${ }^{1,2}$ and exact strip $^{3}$ methods, suitable for use at the preliminary design stage, through to Finite Element (FE) methods ${ }^{4,5}$ that are suitable for use during detailed design. Such techniques make certain assumptions about the loading distribution in the structure, especially when modelling the panel as an individual component, separate from the complete structure, for example the wing box.

The preliminary design program, VICONOPT ${ }^{3}$, considers the panel to be infinitely long (multi-bay) with transverse ribs at longitudinal spacing $l$, thus modelling a panel of length $l$ that is simply supported with warping of the entire cross-section allowed. In addition, uniform end strain is assumed for the whole panel cross-section throughout loading, so that as the skin buckles, and its stiffness is reduced, the point at which the load is applied effectively moves away from the skin to the new (buckled) neutral axis position. As previously shown ${ }^{6}$ these boundary conditions do not arise when using a single bay FE model, simply supported over length $l$. Here, the end load position remains fixed when the skin buckles, so that an offset between load and new neutral axis position causes the panel to always fail on its skin side, regardless of the direction of an overall sinusoidal imperfection; experimental tests ${ }^{6}$ have shown this to be a stable form of failure. The former multi-bay case has an increased stiffener load after skin buckling compared with the single bay case and therefore may lead to stiffener-induced failure of the panel and could be highly unstable ${ }^{7}$. The multi-bay approach is a better representation of an actual aircraft wing-box, where panels are continuous over ribs.

The aim of this paper is to compare the effects of these different boundary conditions on the initial and post-buckling behaviour of a new optimized Jstiffened panel. This was designed using a recently developed version of VICONOPT ${ }^{8,9}$ that estimates the post-buckled stiffnesses of the buckled plates. Comparisons will be made between results given by VICONOPT and a series of ABAQUS ${ }^{4}$ single and double-bay panel models. New strut theory, describing the various load-displacement behaviours before and after initial skin buckling, is first 


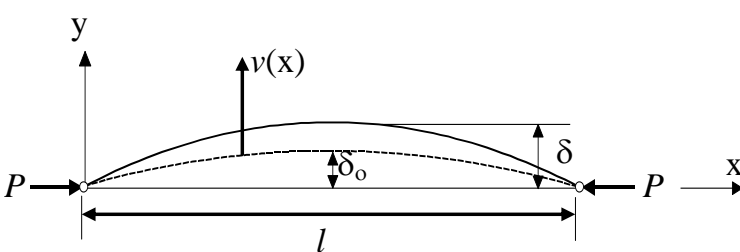

Fig. 1(a). Strut model for initial sinusoidal imperfection $\delta_{\mathrm{o}}$ when $P<P_{s k}$.

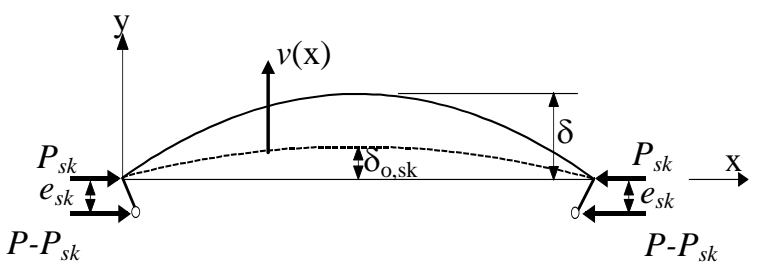

Fig. 1(b). Strut model for initial sinusoidal imperfection $\delta_{\mathrm{o}, \mathrm{sk}}$ and offset load when $P \geq P_{s k}$.

developed for use in VICONOPT and to indicate the difference between the single and multi-bay conditions.

\section{Strut Theory and Shanley Model}

Non-linear, out-of-plane deflections arise at the mid-length of a compressively-loaded panel when either an initial sinusoidal imperfection of amplitude $\delta_{0}$ is present, see Fig. 1(a), or some of the

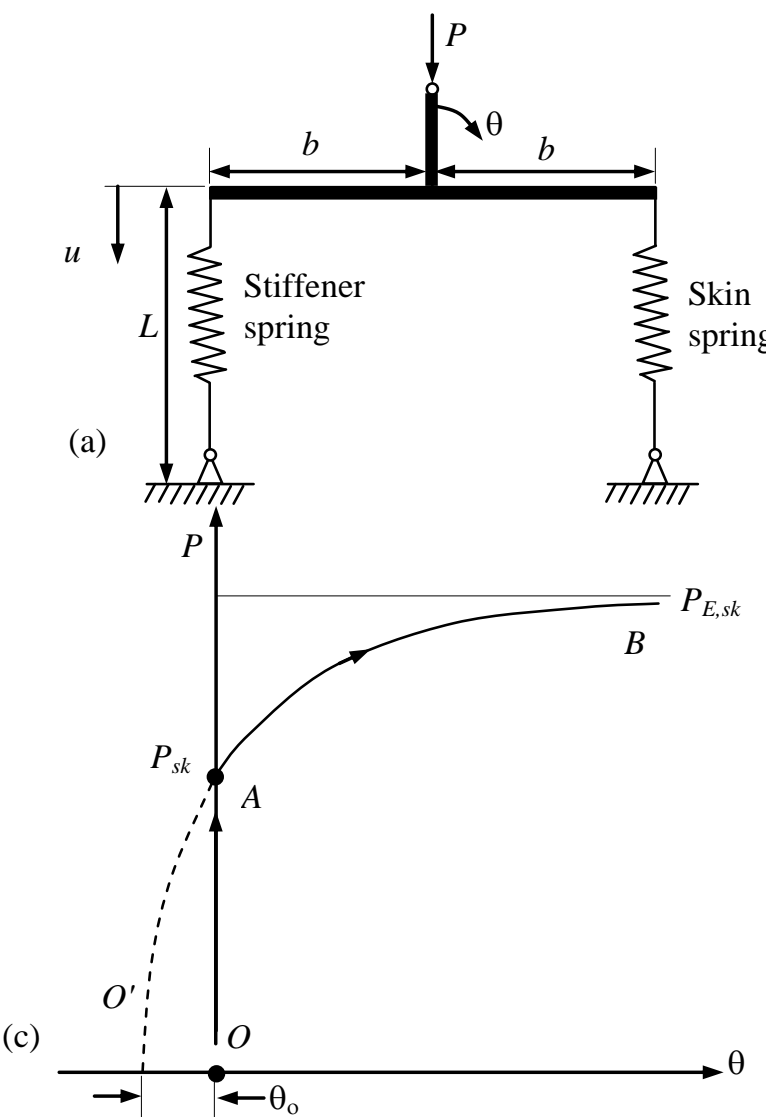

compressive load is offset from the neutral axis of the panel, see Fig. 1(b). Strut theory is used in VICONOPT to calculate these deflections; it is also used in Section 4.5 to compare results for single and multi-bay cases. The basis for this theory follows from the Shanley model, which may be adapted to illustrate the pre- and post- buckling behaviour of stiffened panels ${ }^{10}$. The sudden reduction in stiffness in the skin of a stiffened panel that occurs when a critical skin buckling load is reached also occurs in the Shanley model, where part of the model is made to reduce in stiffness at a critical load. This model is briefly described here to introduce some of the concepts that will later be applied to the strut equations of Section 2.2.

\subsection{Shanley Model}

Fig. 2(a) shows the Shanley model used to represent the stiffened panel, with one spring representing the stiffeners and the other representing the skin. In the case shown here, the system is initially loaded along its neutral axis and no initial imperfection is present. For simplicity, it has been assumed that both skin and stiffener springs initially have stiffness $k$. At a (critical) total load of $P=P_{s k}$, i.e. when the load in the skin is $P_{s k} / 2$, the stiffness of the skin is reduced to $r_{s} k$, to approximate the effect of

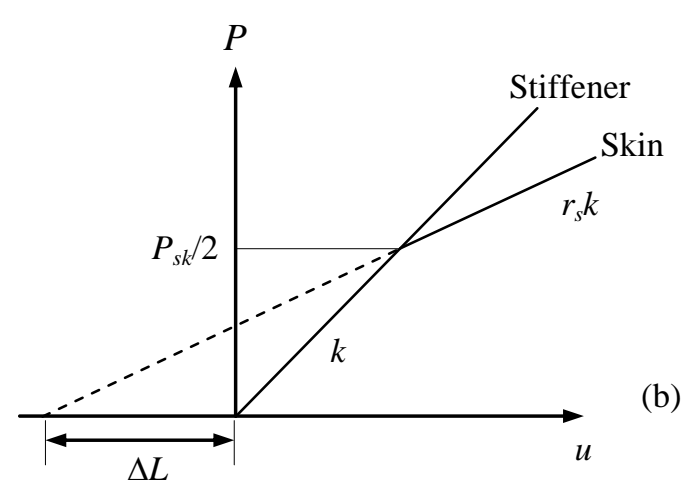

(b)

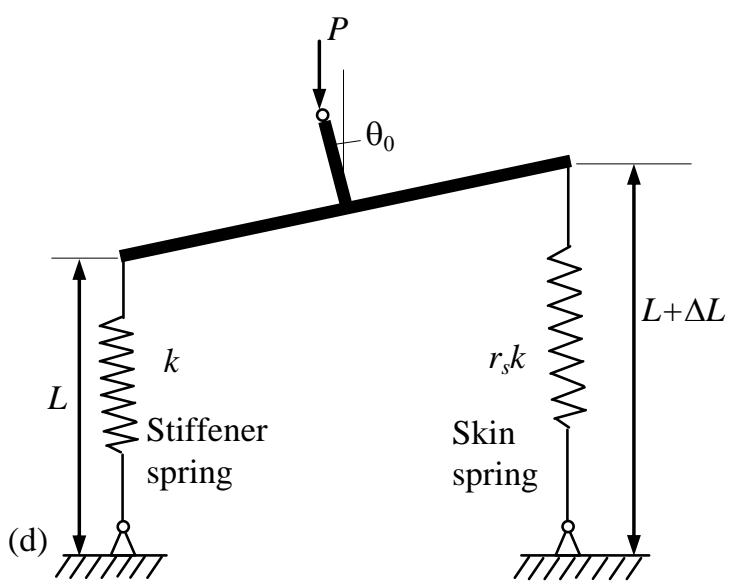

Fig. 2. Shanley model of stiffened panel. (a) Initially perfect case for $P<P_{s k}$ with skin and stiffener stiffness both $k$, see (b), giving equilibrium path $O A$ in (c). (d) Initially imperfect case for $P>P_{s k}$ with skin stiffness reduced to $r_{s} k$, see (b), giving equilibrium path $O^{\prime} A B$ in (c). 
local buckling, see Fig. 2(b). For loads above $P_{s k}$, this reduced stiffness results in rotation of the structure, indicated by $\theta$ in Fig 2(a). Hence, at $P=P_{s k}$, the response of the system switches from equilibrium path $O A$ on Fig. 2(c) to path $A B$. Such a switch introduces an effective negative (initial) imperfection $\theta_{0}$ to the reduced stiffness structure, the value of which depends on both the reduced spring stiffness and the critical load $P_{s k}$. This value of $\theta_{0}$ can be obtained by considering the unloaded system of Fig. 2(d) which has skin stiffness $r_{s} k$, and where $\Delta L$ is the extension required to make the imperfect equilibrium path $O^{\prime} B$ pass through $A$.

One interesting thing to note about the behaviour of this system is that, after the skin reduces stiffness, there is no sudden jump in rotation $\theta$. This may be surprising given that there is a sudden change in the position of the neutral axis, resulting in a sudden offset between it and the position at which the load is applied. However, this view is misleading as it ignores the effect of loading the structure up to $P_{s k}$, and would give rise to a sudden off-loading of the skin at $P_{s k}$. The reduction in stiffness of one of the springs only affects the additional load applied above $P_{s k}$ and thus the rotation of the structure is zero at $P_{s k}$ and smoothly increases with increment of load above $P_{s k}$. This is similar to the case of the stiffened panel, loaded along its original neutral axis both before and after skin buckling. Here there is also a sudden change in stiffness when a critical load is reached and, like the Shanley model, the change in stiffness only affects the extra load applied above the critical load. Hence, using these ideas, strut equations are now developed to form a simplified model of the preand post-buckled behaviour of a stiffened panel.

\subsection{Strut Theory}

For loads below the skin buckling load $P_{s k}$, there is no offset and the mid-length deflection, $\delta$, see Fig. $1(\mathrm{a})$, is given by the following well-known equation,

$$
\delta=\frac{\delta_{\mathrm{o}}}{1-P / P_{E}}
$$

where $P_{E}$ is the Euler or overall buckling load of the unbuckled panel and $\delta_{\mathrm{o}}$ is the amplitude of the initial imperfection.

After the skin has buckled, i.e. $P \geq P_{s k}$, the situation is more complex, and models can be developed to describe single and multi-bay cases. For the single bay case, where the load remains applied at the neutral axis of the original (unbuckled) panel, an offset $e_{s k}$ is introduced to represent the offset associated with the load above $P_{s k}$, see Fig. 1(b). Hence, the following differential equation can be obtained in terms of the vertical deflection $v$ of the strut,

$$
\mathrm{EI} \frac{\mathrm{d}^{2} v}{\mathrm{dx}^{2}}=P_{s k} e_{s k}-P\left[e_{s k}+v+\delta_{\mathrm{o}} \sin \frac{\pi \mathrm{x}}{l}\right]
$$

Solving this for the mid length deflection $\delta$ gives,

$$
\delta=\frac{\delta_{\mathrm{o}, \mathrm{sk}}}{1-\left(P / P_{E, s k}\right)}+e_{s k}\left(1-\frac{P_{s k}}{P}\right)\left(\sec \left(\frac{\pi}{2} \sqrt{\frac{P}{P_{E, s k}}}\right)-1\right)
$$

where $P_{E, s k}$ is the Euler/overall buckling load of the skin-buckled panel. Values of $P_{E, s k}$ and $e_{s k}$ may be calculated by assuming that the Elastic modulus of the skin is reduced by factor $r_{s}$ following buckling. The amplitude of the initial imperfection for the skinbuckled panel $\delta_{\mathrm{o}, \mathrm{sk}}$, which can be thought of as being similar to $\theta_{\mathrm{o}}$ for the above Shanley model, is given by equating Eqs. (1) and (3) with $P=P_{s k}$. Hence,

$$
\delta_{\mathrm{o}, \mathrm{sk}}=\frac{\delta_{\mathrm{o}}}{1-P_{s k} / P_{E}} \times\left(1-\frac{P_{s k}}{P_{E, s k}}\right) .
$$

To model the multi-bay case, where the panel is loaded assuming uniform end shortening both before and after skin buckling so that the load effectively follows the shift in neutral axis as the skin buckles, Eqs. (3) and (4) can still be used, but with $e_{s k}$ set to zero.

In a stiffened panel, the magnitude of the skin buckling load varies with the mid-length deflection arising from the initial imperfection of the panel. The following equation is used to account for this effect,

$$
P_{s k}=\frac{\sigma_{\mathrm{sk}}}{\frac{1}{A}+\frac{\delta y_{s k}}{I}}
$$

where $A$ and $I$ are the cross-sectional area and second moment of area, respectively, of the panel; $y_{s \mathrm{k}}$ is the distance between the neutral axis and skin midthickness of the unbuckled panel and $\sigma_{\mathrm{sk}}$ is the skin buckling stress for the perfect $\left(\delta_{o}=0\right)$ panel. $\sigma_{\text {sk }}$ can be found by using an approximation such as the following well known equation for the buckling of a plate,

$$
\sigma_{s k}=K E\left(\frac{t}{b}\right)^{2}
$$

where $K$ is a suitable constant for the plate boundary conditions, $E$ is the elastic modulus, $t$ is the thickness of the plate and $b$ its width. Alternatively $\sigma_{\text {sk }}$ can be obtained from an analysis of the panel using a program such as VICONOPT. 


\section{VICONOPT Strip Model}

VICONOPT $^{3}$ (VIPASA with CONstraints and OPTimisation) is a FORTRAN 77 program that incorporates the earlier programs VIPASA (Vibration and Instability of Plate Assemblies including Shear and Anisotropy) and VICON (VIPASA and CONstraints). It covers any plate assembly, i.e. panel of constant cross section, composed of anisotropic plates each of which can carry any combination of uniformly distributed and longitudinally invariant inplane stresses. It can be used as either an analysis or an optimum design program. The analysis principally covers the calculations of eigenvalues, i.e., the critical load factors in elastic buckling problems or the natural frequencies in undamped vibration problems. The linear elastic buckling analysis is based upon the exact solution of the governing differential equations of the constituent members, which are assumed to undergo a deformation that varies sinusoidally in the longitudinal direction yielding exact stiffness matrices whose elements are transcendental functions of load factor or frequency and the axial halfwavelength, $\lambda$, of the deformation. The resulting transcendental eigenproblem requires an iterative solution, which is performed using the WittrickWilliams algorithm ${ }^{11}$. The simplest form of the buckling analysis ${ }^{12,13}$ is performed over a range of values of $\lambda$, that usually extends from a value less than the smallest plate width to the length, $l$, of the panel. The lowest buckling load found for any $\lambda$ is taken to be the critical buckling load for the panel. VICONOPT assumes the panel to be an infinitely long (multi-bay) structure made up of portions, which include any supporting structures such as ribs, which repeat at longitudinal intervals $l$. The mode of buckling is assumed to vary sinusoidally in the longitudinal direction with a half-wavelength $\lambda$. In this paper the optimized structure has no applied shear force and is fabricated from an isotropic material so that the nodal lines are necessarily straight and parallel to the panel ends. Also, since $\lambda$ divides exactly into $l$, the boundary conditions are consistent with simple supports at the ends of a panel of length $l$ between ribs. To allow for continuity over the ribs with adjacent bays, warping of the entire crosssection is allowed at the panel end.

For panels designed to have a post-buckling reserve, two modifications ${ }^{14}$ have been made. Firstly, local buckling, considered to be at any $\lambda<l$, is permitted to occur at some prescribed fraction $r_{L}$ of the ultimate design load specified by the user. Secondly, for collapse, which may be due to overall or torsional (stiffener) buckling, the in-plane stiffnesses of the locally buckled plates are reduced by multiplying the pre-buckled stiffnesses by a factor, $r_{s}$, which may be pre-selected by the user ${ }^{6,14}$. However, in this paper, a separate VICONOPT post- buckling analysis ${ }^{9}$ was performed within the optimization procedure so that values of $r_{s}$ were automatically calculated for each plate in the assembly. Each value of $r_{s}$ is obtained by dividing the plate stiffness after buckling by its stiffness before buckling, where each plate stiffness is obtained by dividing the average axial stress in the plate by the total axial strain at the neutral axis of the panel. The axial strain in each plate has two components: one due to axial loading and one due to out of plane flexure. Since the total strain is assumed to be uniform across the panel width, those plates having the greatest strain components due to flexure will have the smallest strain components due to axial load and therefore comparatively small components of axial stress, leading to low values of $r_{s}$.

When a plate is loaded axially and its longitudinal edges are required to remain straight, a non-uniform transverse stress distribution develops. This is composed of tension and compression regions, such that integration along the length gives a zero transverse stress resultant. Because the compression regions occur adjacent to the nodal lines, i.e. lines of zero out of plane displacement, whilst tension regions occur around the mid-length of each half-wavelength, the tension region have a greater stabilizing effect than the destabilizing effects of the compression regions. This is allowed for in VICONOPT $^{9}$ by loading the plate with a uniform (i.e. longitudinally invariant) transverse tension equal to some fraction $\tau$ of the maximum transverse tension calculated from the post-buckling mode. The value $\tau=0.3$ was chosen for the present work, based on previous studies on stiffened panels. ${ }^{8}$

For the VICONOPT analysis, the effects of initial imperfection were calculated using the strut model of Eqs. (1)-(4), with $e_{s k}=0$ so that the load was assumed to follow the shift in the neutral axis. The values for $P_{E}$ and $P_{E, s k}$ were overall panel buckling loads rather than Euler loads.

\section{Example Design}

This section firstly describes the realistic example design problem that has been used to verify the postbuckled design methodology in VICONOPT. The optimum design results produced by VICONOPT are then presented, before comparison with the non-linear finite element program ABAQUS and the strut model described in Section 2.

\subsection{Problem Definition}

The problem used here is based on practical wing data used previously ${ }^{15}$ to demonstrate the application of VICONOPT to optimize panels that are constrained from buckling below thier ultimate loads. For the work presented in the current paper, the 


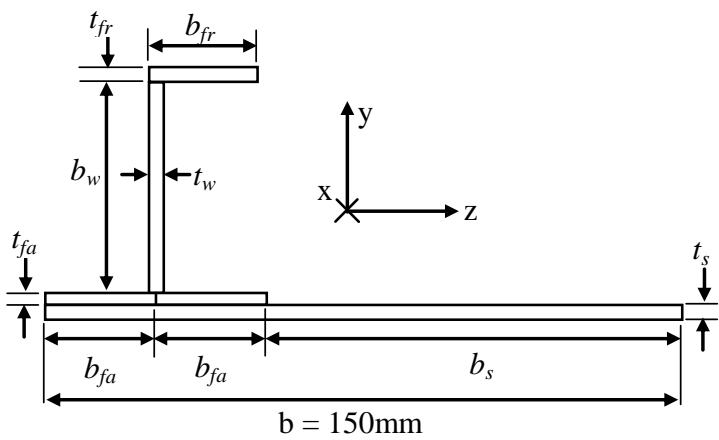

Fig. 3. Cross-section of repeating portion of panel.

design of a single highly loaded compression panel at a location just outboard of the engine has been considered. Here, the ultimate applied end load per unit panel width, due to a $2.5 \mathrm{~g}$ manoeuvre case at cruise speeds with full fuel, is $3.68 \mathrm{kN} / \mathrm{mm}$ and the corresponding limit load is $2 / 3 \times 3.68 \mathrm{kN} / \mathrm{mm}$ $(2.45 \mathrm{kN} / \mathrm{mm})$. To ensure a practical design was obtained a stiffener pitch of $150 \mathrm{~mm}$, panel length of $600 \mathrm{~mm}$ and an inverted $\mathrm{J}$ shaped stiffener were assumed. This geometry is in practice influenced by manufacturing constraints and the values and crosssection chosen are typical for a 150 -seat, twin engine aircraft. The material used, Al7075-T6, had an initial elastic modulus of $74.99 \mathrm{GPa}$, Poisson's ratio of 0.33 and density of $2800 \mathrm{~kg} / \mathrm{m}^{3}$. In the VICONOPT and ABAQUS results that follow, the panel was modelled assuming infinite width.

\subsection{VICONOPT Results}

VICONOPT was used to produce a minimum mass design in which no initial buckling occurred below the limit load, i.e. $r_{L}=2 / 3$, and only stable post-buckled behaviour occurred between limit load and ultimate load. The material was assumed to be linear-elastic.

The panel was modelled as a single repeating portion of width $b=150 \mathrm{~mm}$ with one stiffener and simply supported ends, as shown in Fig. 3. The model allowed for offsets between the centre-lines of connected plates ${ }^{12}$. The panel was designed with VICONOPT assuming a negative imperfection $\delta_{0}=-l / 1000$ which increases compression in the stiffener relative to the skin. Table 1 lists the seven independent design variables $b_{f r}, b_{w}, b_{f a}, t_{f r}, t_{w}, t_{f a}$ and $t_{s}$. The breadth of the unsupported skin was a dependent variable $b_{s}=b-2 b_{f a}$. The cross-sectional area of one stiffener is defined as $A_{s}=b_{f r} t_{f r}+b_{w} t_{w}+2 b_{f a} t_{f a}$ and the area of skin within one repeating portion equals $b t_{s}$. The ratio $\gamma\left(=A_{s} / b t_{s}\right)$ and mass of one repeating portion are also listed in Table 1. Twenty design cycles were performed but the panel mass had converged to within $2 \%$ of its final value after just 4 cycles.

Table 1. Starting and final values of designed panel.

\begin{tabular}{c|ccc}
\hline \hline Variable & $\begin{array}{c}\text { Constraint } \\
\text { Value }\end{array}$ & Start & End \\
\hline$b_{f r}, \mathrm{~mm}$ & $\geq 25$ & 40 & 30.26 \\
$b_{w}, \mathrm{~mm}$ & $40 \leq b_{w} \leq 65$ & 65 & 49.02 \\
$b_{f a}, \mathrm{~mm}$ & $25 \leq b_{f a} \leq 45$ & 30 & 36.09 \\
$b_{s}, \mathrm{~mm}$ & $=150-2 b_{f a}$ & 90 & 77.83 \\
$t_{f r}, \mathrm{~mm}$ & $\geq 0.5$ & 5 & 3.95 \\
$t_{w}, \mathrm{~mm}$ & $\geq 0.5$ & 3 & 4.73 \\
$t_{f a}, \mathrm{~mm}$ & $\geq 0.5$ & 3 & 1.42 \\
$t_{s}, \mathrm{~mm}$ & $\geq 0.5$ & 5 & 4.06 \\
$\gamma=A_{s} / b t_{s}$ & - & 0.77 & 0.75 \\
\hline Mass, kg & - & 2.23 & 1.78 \\
\hline \hline
\end{tabular}

Although the design was carried out without explicit constraints on strain or the value of the ratio $\gamma$, analysis of the final panel configuration confirmed that all strains remained below the assumed elastic limit of $6770 \mu$ strain at the limit load and that $\gamma$ remained in the range $0.75 \leq \gamma \leq 1.00$ which is typically acceptable in industry. The resulting design also met all the design requirements when modelled as perfect or with a positive imperfection, $\delta_{\mathrm{o}}=+l / 1000$.

Table 2. Summary of VICONOPT and ABAQUS results for the final VICONOPT design showing: initial buckling loads (and associated half-wavelengths) obtained using linear and non-linear analyses, post-buckled collapse loads, collapse modes, mid-surface strains at limit load $\left(\varepsilon_{\text {limit }}\right)$ and post to pre buckling stiffness ratios $\left(k^{*} / k\right)$ of the whole panel. Note: SK, U, and ST denote skin, uniform and stiffener respectively.

\begin{tabular}{|c|c|c|c|c|c|c|c|}
\hline \multirow[t]{2}{*}{ Model } & \multirow[t]{2}{*}{$\delta_{o}$} & \multicolumn{2}{|c|}{ Initial buckling(kN/mm) } & \multicolumn{2}{|c|}{ Collapse } & \multirow{2}{*}{$\begin{array}{c}\varepsilon_{\text {limit }} \\
(\mu \text { strain })\end{array}$} & \multirow[t]{2}{*}{$k^{*} / k$} \\
\hline & & Linear & Non-linear & $(\mathrm{kN} / \mathrm{mm})$ & Mode & & \\
\hline \multirow{3}{*}{ VICONOPT } & $+l / 1000$ & $2.48(l / 5)$ & - & 3.73 & Overall/skin & $4800(\mathrm{SK})$ & 0.68 \\
\hline & 0 & $2.59(l / 5)$ & - & 3.79 & Overall & $4600(\mathrm{U})$ & 0.69 \\
\hline & $-l / 1000$ & $2.73(l / 5)$ & - & 3.65 & Torsional & $5300(\mathrm{ST})$ & 0.70 \\
\hline ABAQUS & $+l / 1000$ & - & $2.48(l / 5)$ & $\approx 3.52$ & Overall/skin & $5010(\mathrm{SK})$ & 0.53 \\
\hline \multirow[t]{2}{*}{ Single bay } & 0 & $2.63(l / 5)$ & $2.60(l / 5)$ & $\approx 3.57$ & Overall/skin & $4930(\mathrm{SK})$ & 0.54 \\
\hline & $-l / 1000$ & - & $2.70(l / 5)$ & 3.63 & Overall/skin & $5390(\mathrm{ST})$ & 0.55 \\
\hline ABAQUS & $+l / 1000$ & - & $2.51(l / 5)$ & 3.66 & Overall/skin & $4893(\mathrm{SK})$ & 0.56 \\
\hline \multirow[t]{2}{*}{ Double bay } & 0 & $2.60(l / 5)$ & $2.61(l / 5)$ & 3.79 & Overall/skin & $4823(\mathrm{SK})$ & 0.63 \\
\hline & $-l / 1000$ & - & $2.65(l / 5)$ & 3.71 & Torsional/overall & $5542(\mathrm{ST})$ & 0.64 \\
\hline
\end{tabular}


The first part of Table 2 lists the VICONOPT analysis results for the final panel configuration. For all the imperfection cases the initial buckling mode is a local mode $(\lambda=l / 5)$, the collapse load is near or above the design requirement of $3.68 \mathrm{kN} / \mathrm{mm}$ and the peak strains at limit load $\left(\varepsilon_{\text {limit }}\right)$ are elastic. These peak strains are measured at the mid-surface of the plate in which the strain occurs. For a positive imperfection $\left(\delta_{\mathrm{o}}=+l / 1000\right)$ VICONOPT predicts collapse at $3.73 \mathrm{kN} / \mathrm{mm}$ in an overall/skin mode. For a negative imperfection $\left(\delta_{0}=-l / 1000\right)$ VICONOPT predicts collapse at $3.65 \mathrm{kN} / \mathrm{mm}$ in a torsional mode which will precipitate overall failure. For zero imperfection VICONOPT predicts collapse at $3.79 \mathrm{kN} / \mathrm{mm}$ in an overall mode. The last column of the table lists the ratios of post-buckled to prebuckled stiffness, $k^{*} / k$, for the panel. The corresponding values of $r_{s}$ for each plate are listed in Table 3. It can be seen that the values for locally buckled skin plates are lower than would be obtained by considering a plate with similar boundary conditions in isolation for the reasons outlined in Section 3 .

Table 3. Final panel configuration $r_{s}$ values for each plate.

\begin{tabular}{c|cccc}
\hline \hline$\delta_{\mathrm{o}}$ & $\begin{array}{c}\text { Remote } \\
\text { flange }\end{array}$ & Web & $\begin{array}{c}\text { Attached } \\
\text { flange }\end{array}$ & Skin \\
\hline$+l / 1000$ & 0.99 & 0.98 & 0.87 & 0.10 \\
0 & 0.99 & 0.98 & 0.87 & 0.13 \\
$-l / 1000$ & 0.99 & 0.98 & 0.88 & 0.17 \\
\hline \hline
\end{tabular}

\subsection{Finite Element Modelling}

FE modelling was carried out using the package $\mathrm{ABAQUS}^{4}$ to determine both the linear (bifurcation) and non-linear ${ }^{16}$ buckling behaviour allowing comparison of the single and double-bay FE models with VICONOPT and strut model results. Two FE models were developed, both using QUAD4 (S4R5) thin shell elements. In order to approximate a panel of infinite width only 2 repeating portions were modelled. The $\delta_{\mathrm{x}}, \delta_{\mathrm{y}}, \theta_{\mathrm{x}}, \theta_{\mathrm{y}}$ and $\theta_{\mathrm{z}}$ degrees of freedom at each node along one longitudinal edge (e.g. node $\mathrm{A}$ of Fig. 4) were set equal to corresponding degrees of freedom of the equivalent node on the other longitudinal edge of the model, e.g. node B. For the results of Sections 4.4.1 - 4.4.4 the effects of material plasticity were not considered.

\subsubsection{Single Bay Model}

The single bay model used 4800 elements and was similar to the one given in Ref. 6. This modelled the panel using a single longitudinal portion of length $l$. The panel was simply supported at the ends with the axis about which the end cross-section of the panel rotates coincident with the neutral axis of the unbuckled panel. The cross-sectional geometry of skin and stiffener at the ends of the panel was prevented from deforming using multi-point constraints. Axial load was applied using point loads distributed so that the effective load axis of the panel was along the neutral axis of the unbuckled system. When the skin buckled the load remained applied at the neutral axis of the unbuckled panel. The panel was analysed to obtain the bifurcation buckling behaviour of a perfect panel and the non-linear behaviour of both perfect and imperfect panels. The imperfect cases used a sinusoidal overall imperfection of amplitude $\delta_{\mathrm{o}}= \pm l / 1000$.

\subsubsection{Double Bay Model}

In this case a 9600 element model consisting of a portion of panel of length $2 l$ was created. As shown in Fig. 4, ribs, comprising of an additional 420

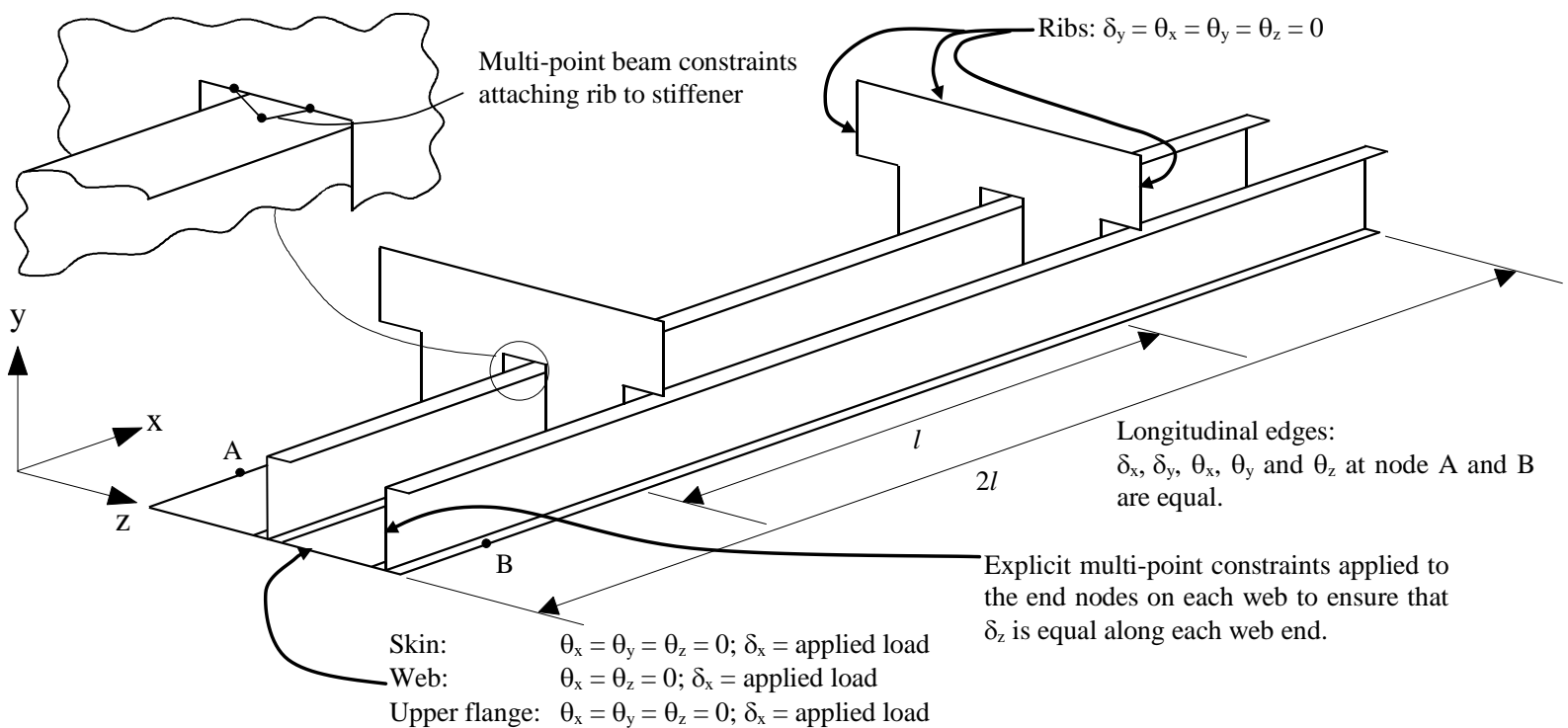

Fig. 4. Double-bay FE model showing rib and panel boundary condition and longitudinal edge conditions to approximate an infinite width panel. 
QUAD4 (S4R5) thin shell elements were attached to the panel skin at $\mathrm{x}=0.5 \mathrm{l}$ and $1.5 \mathrm{l}$. Cut-outs in the ribs allowed the stiffeners to pass freely although as shown in the enlarged section in Fig. 4, each stiffener was connected to a rib using two rigid multi-point constraints. This represents the cleat used in practice to attach the rib to the upper flange of the stiffener. The side and upper edges of the ribs had clamped boundary conditions, with displacements in the $\mathrm{x}$ and $\mathrm{z}$ directions allowed. At the ends of the panel the boundary conditions depicted in Fig. 4 were imposed. Axial load was applied using uniform end strain in the $\mathrm{x}$ direction at one end of the panel, with displacement in the $\mathrm{x}$ direction restrained at the other end. This resulted in load being applied along the neutral axis of the panel before skin buckling and at the new neutral axis position after skin buckling. The stiffener webs at the panel ends were restrained to remain vertical using multi-point constraints. The $\delta_{z}$ degree of freedom was not suppressed at the ribs or panel ends so that the buckling and collapse loads would not be reduced due to the Poisson's ratio effect. The panel was analysed to obtain the bifurcation buckling behaviour of a perfect panel and the nonlinear behaviour of both perfect and imperfect panels. The imperfect cases used a global imperfection that consisted of a complete cosine wave, of amplitude $\delta_{\mathrm{o}}$, along the length of the panel $2 l$. When examining the results only the behaviour of the portion of length $l$ between the ribs was considered and a positive imperfection increases the relative compression in the skin compared with the stiffener for this portion.

\subsection{FE Results}

\subsubsection{Initial Buckling}

From the initial buckling loads given in Table 2 it can be seen that both FE models, using linear analysis, give slightly higher initial buckling loads for the perfect panel compared to the VICONOPT solution, with the double bay model giving the closer result. This may be due to the FE method slightly overestimating the stiffness of the panel. Both FE models predict that the panel will initially buckle with 5 half-wavelengths in the skin which agrees with the VICONOPT solution.

The non-linear estimates of the initial buckling load for the two FE models, of the perfect case, as seen in Table 2, are again slightly higher than predicted by VICONOPT. The initial buckling mode predicted is a skin mode with 5 half-wavelength, matching both the VICONOPT and ABAQUS linear solutions. However unlike the linear FE analysis the single bay solution now gives the best estimate, being within $0.4 \%$ of the VICONOPT solution. The double bay non-linear initial buckling solution is almost identical to that given by the linear analysis.
For the imperfect cases it can be seen in Table 2 that there is good agreement between the VICONOPT and the FE non-linear solutions for initial buckling, except in the case of the $-l / 1000$ solution for the ABAQUS double bay mode. Here ABAQUS predicts an initial buckling load that is about 3\% lower than the VICONOPT prediction. It is thought that this is due to the shape of the negative imperfection applied to the double bay model. This increases the compression in the skin at the ends of the panel, outside the ribs, so that these skin sections buckle early inducing buckling of the centre section before it would otherwise occur. For all the linear and non-linear FE analyses, the initial buckling load predicted is always greater than the initial buckling load requirement of $2.45 \mathrm{kN} / \mathrm{mm}$.

\subsubsection{Collapse}

The ABAQUS double bay solutions indicate that the panel design reaches the design load $(3.68 \mathrm{kN} / \mathrm{mm})$ before collapse occurs, with the exception of the $+l / 1000$ case, which can carry a load within $0.54 \%$ of the design load. The failure modes obtained by both the VICONOPT and ABAQUS double bay solutions are similar. For the $+l / 1000$ and the perfect cases both predict that failure is due to an overall mode. With the exception of the VICONOPT perfect case this failure mode also involves a stable interaction with the local skin buckling mode of the type shown in Fig. 5. For the $-l / 1000$ case both methods indicate that failure is due to an $l / 2$ torsional mode, as shown in Fig. 6, which is potentially more
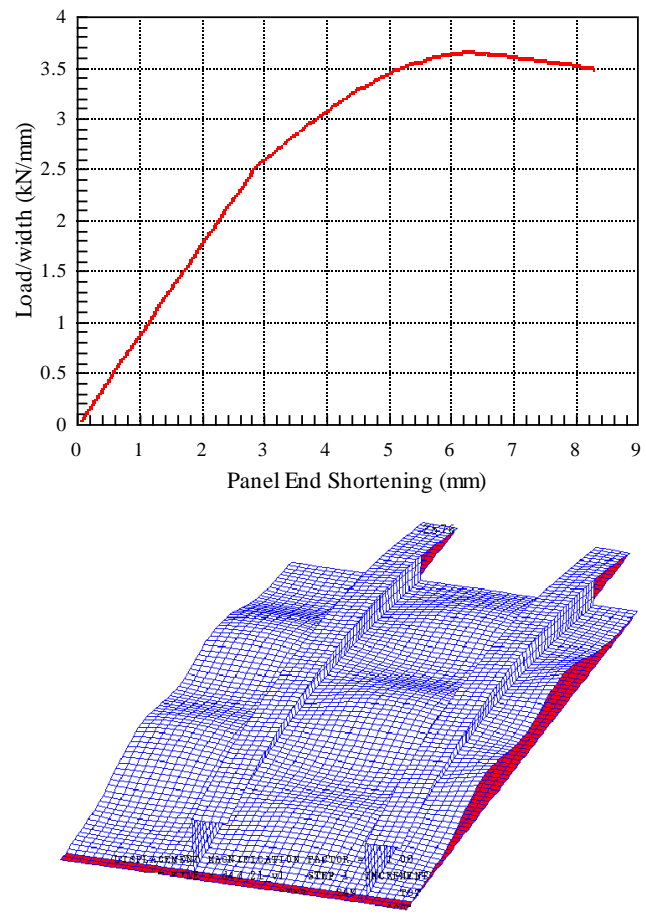

Fig. 5. End-shortening plot and final deformed shape given by ABAQUS double bay model for the $+l / 1000$ case. 

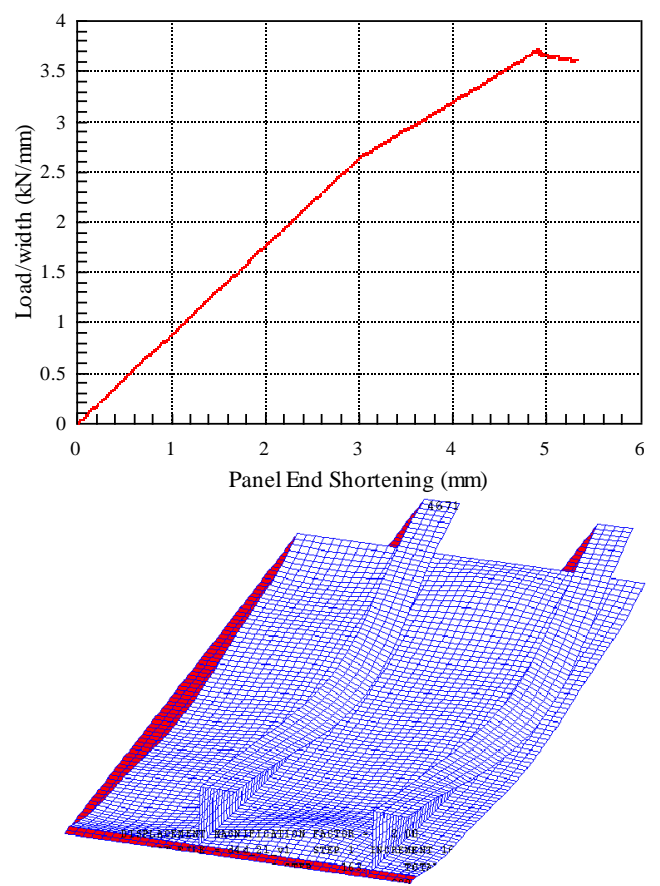

Fig. 6. End-shortening plot and final deformed shape given by ABAQUS double bay model for the $-l / 1000$ case.

serious as this type of failure can be unstable.

Conversely, the ABAQUS single bay model, for all cases, predicts failure modes which are a stable interaction between the local skin and the overall buckling modes, similar to that shown in Fig. 5. This is due to the offset between the load and neutral axes that occurs when the skin buckles, as discussed in Section 1. The effect of the offset, for the $-l / 1000$ case, on the out of plane displacement of the single bay model compared to the double bay model can clearly be seen in Fig. 7. These results were obtained from the average vertical displacement of the 2 midlength nodes closest to the neutral axis of the unbuckled panel on each stiffener in the model. The FE single bay solutions indicate that the design load

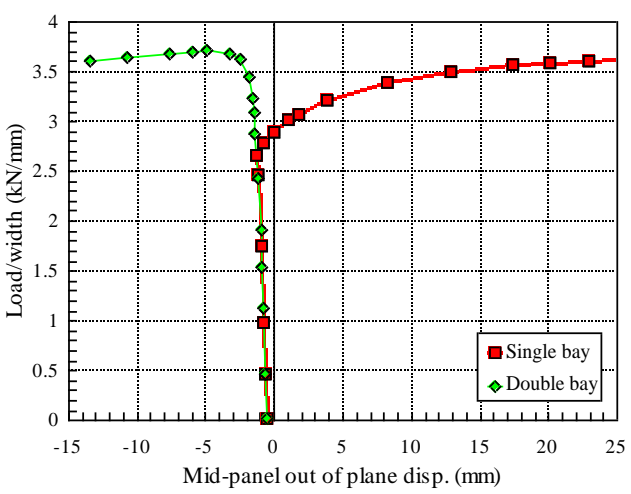

Fig. 7. Mid-length out of plane displacement, $\delta$, for the ABAQUS single and double bay models, illustrating the effect of the offset for the $-l / 1000$ case. is not reached for any imperfection case, due to this effect. As with the double bay model, the $+l / 1000$ case has the lowest collapse load, $4.2 \%$ below the design load. It should be noted that it is hard to determine the exact collapse loads for the perfect and $+l / 1000$ cases as the solution was becoming asymptotic to the overall load of the reduced stiffness panel for these cases.

\subsubsection{Limit Strains}

The mid-thickness peak strains predicted by ABAQUS at approximately the limit load are also given in Table 2. It should be noted that it is impossible to obtain the strains in the ABAQUS solution at exactly the limit load and therefore the strains have been given for the non-linear step which is at the load closest to the limit load. It can be seen that strain levels in the panel predicted by ABAQUS at mid-thickness match reasonably well the strain levels predicted by VICONOPT. Since strain is being measured at a load level where the effect of the offset in the ABAQUS single bay model, has little effect, the positions in the panel where the peak strains occur are the same for both models.

\subsubsection{Stiffness Ratio}

From Table 2 it can be seen that the predictions of the panel stiffness ratio $\left(k^{*} / k\right)$ given by VICONOPT and ABAQUS do not match very well. The ABAQUS predictions are significantly lower that those given by VICONOPT. This may be due to the value of the transverse tension factor $\tau$ that has been used for the VICONOPT post-buckling analysis. Analysis of a previous design has shown that changing $\tau$ can have a significant affect on the value of $k^{*} / k$ obtained, although this may also effect the predicted values of the collapse loads.

\subsubsection{Material Plasticity}

The effects of material plasticity were briefly investigated in the ABAQUS double bay model by including an experimentally derived stress-strain relationship for A17075-T6 in the FE model. It was found that this gave initial buckling loads of $2.56 \mathrm{kN} / \mathrm{mm}$ and $2.61 \mathrm{kN} / \mathrm{mm}$ for the perfect and $-l / 1000$ cases, respectively. In both cases initial buckling was in a skin mode consisting of 5 halfwavelengths. The biggest effect of the material plasticity was on the collapse loads, which for the two cases considered occurred simultaneously with initial buckling. The reason for this will be described in Section 5.

\subsection{Strut Model Results}

Results for the final VICONOPT optimum design are presented in this section. The value of $e_{s k}$ used in the strut model of the single bay case was obtained assuming the elastic modulus of the unsupported skin 
of the panel was multiplied by the factor $r_{s}=0.133$ when the unsupported skin, width $b_{s}$, buckled. This gave an offset between the neutral axes of the full and reduced stiffness panels of $4.334 \mathrm{~mm}$. The value, 0.133 , is the average of the skin stiffness reduction values given in Table 3 . The overall buckling loads for the full and reduced stiffness panels, $P_{E}$ and $P_{E, s k}$ respectively, were obtained using VICONOPT.

Figure 8 shows the mid-length out of plane displacement verses load behaviour given by Eqs. (1) and (3) for the single bay strut model and compares this solution with the behaviour predicted by the single bay FE model. As can be seen, there is good agreement between the strut and FE models for the negative, perfect and positive imperfections. The results confirm that failure is in the positive $\delta$ direction, i.e. in an overall/skin mode in all cases. Figure. 9 compares the mid-length out of plane displacement verses load behaviour given by the strut and FE models for the double bay case. Here, it can be seen that there are significant differences in the out of plane displacement behaviour predicted by the two methods after skin buckling. However, both methods do, for the $-l / 1000$ case, predict a failure on the stiffener side. The FE model solutions always have a more positive amplitude at a given load level than the strut model results; thus the skin is in greater compression in the FE model. An additional small offset term, to account for this difference, was then added to the strut model for loads above $P_{s k}$. Figure 10 shows that using a value of $e_{s k}=1.1 \mathrm{~mm}$ gives good agreement with the FE solution. It has also been shown for this design that adding an addition overall imperfection term for loads above $P_{s k}$ can instead be used to correct the differences seen in Fig. 9.

\section{Concluding Remarks}

VICIONOPT has been used to design a panel for which skin buckling occurs at $68 \%$ of ultimate load. The assumptions made represent multi-bay aerospace applications, in the sense that the panel is continuous over rib supports, and load is applied via uniform end shortening.

Comparisons of the (elastic) ABAQUS double bay model and the VICONOPT results indicate agreement of better than $3 \%$ on initial buckling loads and $2 \%$ on collapse loads, despite poor correlation between the post-buckled stiffnesses of the two models. The panel has been shown by VICONOPT to have a stiffener buckling failure mode when an overall sinusoidal imperfection causing increased stiffener compression is present. Such a failure is not seen in a typical ABAQUS single bay model of such panels. However, the failure is confirmed by the double bay model.

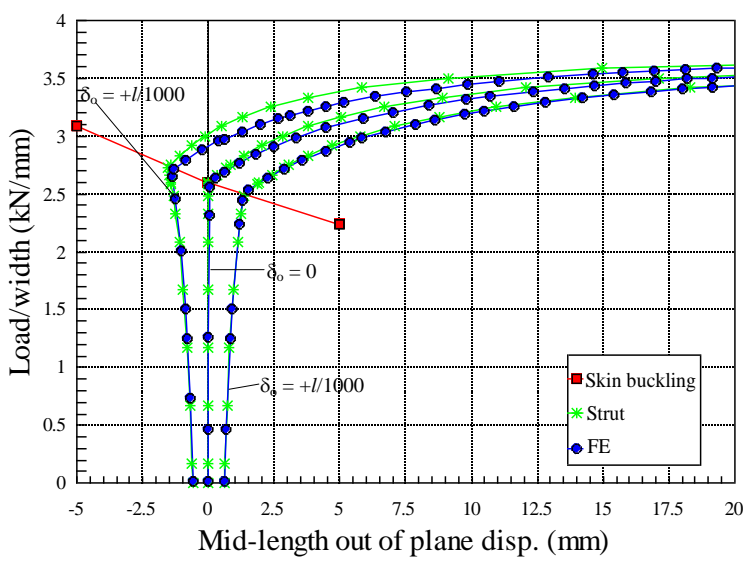

Fig. 8. Mid-length out of plane displacement, $\delta$, for the single bay ABAQUS and strut models.

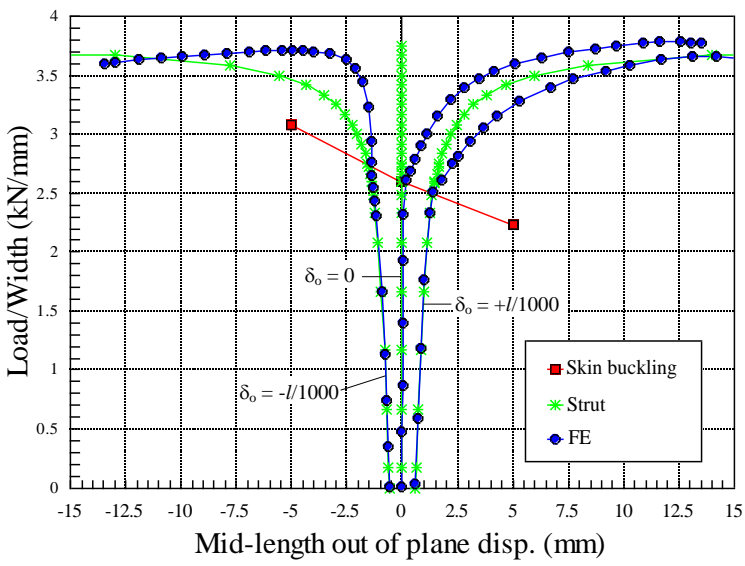

Fig. 9. Mid-length out of plane displacement, $\delta$, for the double bay ABAQUS and strut models.

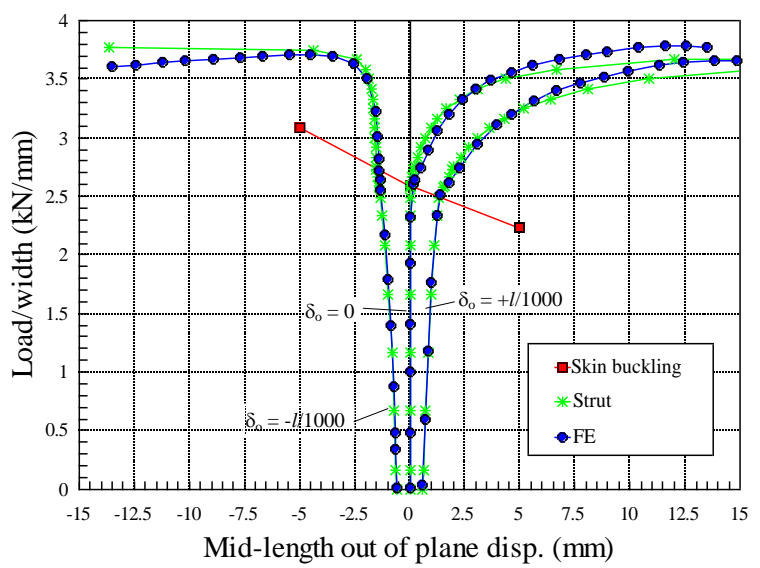

Fig. 10. Mid-length out of plane displacement, $\delta$, for double bay ABAQUS model and strut model with $e_{s k}$ $=1.1 \mathrm{~mm}$.

It is important to note that an ABAQUS double bay model, accounting for material non-linearity, indicated that the panel would actually have little or no post-buckled reserve of strength, collapsing at around $69 \%$ of the ultimate design load. Hence one might conclude that VICONOPT is currently not able to produce a reliable design for such a realistic example. Whilst this is true for the problem chosen, which is heavily loaded and has close values of initial 
buckling load and material yield load, a more lightly loaded panel would not be expected to present such proximity in loads. Such a panel would be designed with a thinner skin: since stiffener spacing is generally kept fixed along the wing, skin buckling load is directly proportional to the cube of skin thickness. Thus the skin buckling load for such a panel would be substantially lower than its yield load, which is directly proportional to skin thickness. A more lightly loaded panel would therefore be expected to exhibit linear material behaviour well into the post-buckled range. Nevertheless, it is important to warn designers of the dangers of ignoring material non-linearity when optimizing heavily loaded panels.

Finally, the paper has shown that a newly developed strut model can be used to represent the out-of-plane deflection of axially loaded, postbuckled panels. The model shows good agreement with ABAQUS single bay results. It also indicates the presence of an additional imperfection in the double bay ABAQUS model, which may be represented by offsetting the load towards the skin in the strut model.

\section{Acknowledgements}

The authors wish to thank the Engineering and Physical Sciences Research Council (GR/M26200 and GR/M26237) and Airbus UK Ltd for providing the financial assistance that made this work possible. In addition they would like to thank Prof. F.W. Williams and Dr. M.S. Anderson for their helpful comments.

\section{References}

1. Dawe, D.J., Lam, S.S.E, and Azizian, Z.G., "Finite Strip Post-Local-Buckling Analysis of Composite Prismatic Plate Structures," Computers and Structures, Vol. 48, 1993, pp. 1011-1023.

2. Bushnell, D., "PANDA2 - Program for minimum weight design of stiffened, composite, locally buckled panels, " Computers \& Structures, Vol. 25, 1987, pp. 469-605.

3. Butler, R., and Williams, F.W., "Optimum Design Using VICONOPT, a Buckling and Strength Constraint Program for Prismatic Assemblies of Anisotropic Plates," Computers and Structures, Vol. 43, 1992, pp. 699-708.

4. Hibbitt, Karlsson, and Sorensen, Inc., "ABAQUS/Standard User's Manual," Version 5.8, Pawtucket, RI, 1999.

5. ANSYS User's manual, Version 5.0, Swanson Analysis Systems Inc., Houston, PA, 1992.

6. Lillico, M., Butler, R., Hunt, G.W., Watson, A., Kennedy, D., and Williams, F.W., "Optimum Design and Testing of a Post-Buckled Stiffened
Panel," Proceedings of the $41^{\text {st }}$ AIAA/ASME/ ASCE/AHS/ASC Structures, Structural Dynamics and Materials Conference, Atlanta, GA, AIAA2000-1659.

7. Butler, R., Lillico, M., Hunt, G.W., and McDonald, N.J., "Experiments on Interactive Buckling in Optimized Stiffened Panels," Proceedings of the $1^{\text {st }}$ ASMO UK/ISSMO Conference on Engineering Design Optimization, Ilkley, U.K., 1999, pp. 89-96.

8. Anderson, M.S., "Inclusion of Local PostBuckling Response in the design of Stiffened Panels," Proceedings of the 41st AIAA / ASME/ ASCE/AHS/ASC Structures, Structural Dynamics and Materials Conference, Atlanta, GA, 2000, AIAA-2000-1661.

9. Powell, S.M, Williams, F.W, Askar, A.-S., and Kennedy, D., "Local Postbuckling Analysis for Perfect and Imperfect Longitudinally Compressed Plates and Panels," Proceedings of the 39th AIAA/ASME/ASCE/AHS/ASC Structures, Structural Dynamics and Materials Conference, Long Beach, CA, 1998, pp. 595-603.

10. Thompson, J.M.T, Tulk, J.D., and Walker, A.C., "An Experimental Study of ImperfectionSensitivity in the Interactive Buckling of Stiffened Panels," Buckling of Structures, edited by B. Budiansky, Springer-Verlag, Berlin, Germany, 1976, pp.149-159.

11. Wittrick, W.H., and Williams, F.W., "A General Algorithm for Computing Natural Frequencies of Elastic Structures," Quarterly Journal of Mechanics and Applied Mathematics, Vol. 24, Part 3, 1971, pp. 263-284.

12. Wittrick, W. H., and Williams, F. W., "Buckling and Vibration of Anisotropic or Isotropic Plate Assemblies Under Combined Loadings," International Journal of Mechanical Sciences, Vol. 16, 1974, pp. 209-239.

13. Anderson, M. S., Williams, F. W., and Wright, C. J., "Buckling and Vibration of Any Prismatic Assembly of Shear and Compression Loaded Anisotropic Plates with an Arbitrary Supporting Structure," International Journal of Mechanical Sciences, Vol. 25, 1983, pp. 585-596.

14. Anderson, M.S., "Design of Panels Having Postbuckling Strength," Proceedings of 38th AIAA/ASME/ASCE/AHS/ASC Structures, Structural Dynamics, and Materials Conference, Kissimme, FL, 1997, pp. 2407-2413.

15. Butler, R. "Optimum Design of Composite Stiffened Wing Panels-A Parametric Study", Aeronautical Journal, May 1995, pp. 169-177.

16. Riks, E., "An Incremental Approach to the Solution of Snapping and Buckling Problems", International Journal of Solids and Structures, Vol. 15, 1979, pp. 529-551. 
